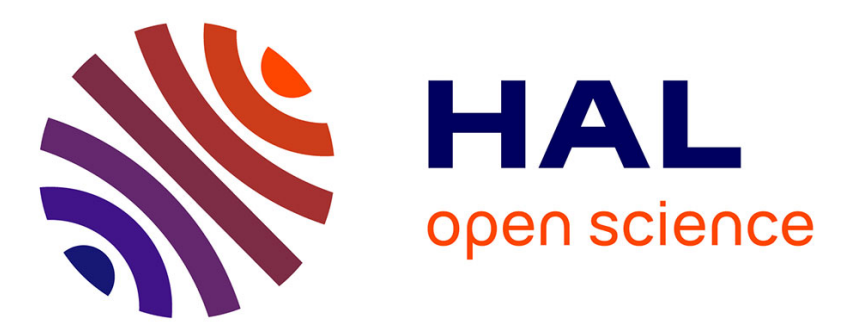

\title{
Microstructure Inspection by Means of Mechanical Barchausen Effect Analysis
}

\author{
B. Augustyniak, J. Degauque
}

\section{To cite this version:}

B. Augustyniak, J. Degauque. Microstructure Inspection by Means of Mechanical Barchausen Effect Analysis. Journal de Physique IV Proceedings, 1996, 06 (C8), pp.C8-527-C8-530. 10.1051/jp4:19968114 . jpa-00254544

\section{HAL Id: jpa-00254544 https://hal.science/jpa-00254544}

Submitted on 1 Jan 1996

HAL is a multi-disciplinary open access archive for the deposit and dissemination of scientific research documents, whether they are published or not. The documents may come from teaching and research institutions in France or abroad, or from public or private research centers.
L'archive ouverte pluridisciplinaire HAL, est destinée au dépôt et à la diffusion de documents scientifiques de niveau recherche, publiés ou non, émanant des établissements d'enseignement et de recherche français ou étrangers, des laboratoires publics ou privés. 


\title{
Microstructure Inspection by Means of Mechanical Barchausen Effect Analysis
}

\author{
B. Augustyniak and J. Degauque* \\ Department of Applied Physics and Technical Matematics, Technical University of Gdansk, \\ 80-355 Gdánsk, Poland \\ * Department of Physics, INSA de Toulouse, 31520 Toulouse cedex, France
}

\begin{abstract}
This paper presents results of the application of the mechanical Barkhausen effect (MBE) for the evaluation of the strain dependence of magnetomechanical intemal friction (MIF) and the intemal stress distribution function. Both functions were obtained from the "first load" mechanical Barkhausen effect (MBE) strain dependence. The MBE intensity was measured with torque motor applying shear stress with constant time rate. This new method was tested for pure iron and Fe-C (160 ppm of C) alloy with different precipitation states. The evaluated strain dependence of the MIF was compared with the experimental MIF functions measured with a pendulum. It is sugested that the very close relationship between the two dependencies confirms the validity of the performed evaluation of the internal stress distribution function.
\end{abstract}

\section{INTRODUCTION}

External stress induces motion of 'non-180 magnetic domain walls (DW) leading to the magnetomechanical internal friction (MIF) of vibrating samples $[1,2,3]$. The abrupt motion of DW provides the change of the magnetic flux intensity which can be detected by a pick-up coil. The induced voltage pulses depicts the mechanical Barkhausen effect (MBE) signal [4,5]. It was revealed that the MBE is a new source of quantitative information about magneto-mechanical damping. Very close relation of the MBE intensity with the magneto-mechanical energy losses was confirmed by the linear dependence between integral over one cycle of MBE intensity hysteresis and energy losses in one cycle as calculated from the MIF [6,7]. Using such dependence one can evaluate the internal stress distribution function $N(\sigma)$ diretly from the MBE stress dependce. The $N(\sigma)$ function provides substantial information about nature and source of local stress barriers and is used for the calculation of the fundamental parameter $\sigma_{i}$ the mean value of internal stresses. That function is actually or arbitrary proposed to match experimental stress dependence of MIF $[2,8]$ or is evaluated from that MIF stress dependence by appropriate differentiating [3]. The first method is self consistent but depends on the chosen model of hysteresis process. The second method leads to some reasonable estimation of that function but without any information about energy losses distribution during cycling. The stress dependence of the MBE intensity has been used successfully to internal stress distribution function evaluation [9] but using the $N(\sigma)$ functions proposed by Birchak and Smith [2]. The aim of the paper is to present a new approach to the MBE intensity analysis which leads to the evaluation of the MIF strain dependence and the internal stress distribution function. It is proposed to evaluate both dependencies from the same MBE intensity stress dependence function which is measured during the "first load". 


\section{EXPERIMENTAL RESULTS}

\subsection{Samples}

We have investigated the $\mathrm{Fe}-\mathrm{C}(160 \mathrm{ppm}$ of $\mathrm{C}$ ) alloys with different internal stress distributions due to the carbide precipitates evolution [10]. There was also tested a pure iron annealed sample. The Fe-C alloys have been prepared from pure iron by means of zone melting method using induction furnace and levitation-like way of the material suspention. Carbonation was performed by doping the liquid iron phase with graphite. The cast was laminated at room temperature to obtain sheets of about $0.2 \mathrm{~mm}$ thick from which the plates $80 \mathrm{~mm}$ long and $10 \mathrm{~mm}$ large were prepared. The samples have the form of plates with $0.4 \mathrm{~mm}$ thickness. The pure iron sample (state labeled 'Fel') was annealed at $1050 \mathrm{~K}$ for $5 \mathrm{~h}$ in vacuum. The $\mathrm{Fe}-\mathrm{C}$ samples were annealed for $1 \mathrm{~h}$ at $990 \mathrm{~K}$ and quenched in water of $273 \mathrm{~K}$ to obtain a homogenious solution of $\mathrm{C}$ (state labeled as ' $\mathrm{s}$ ). The $\mathrm{Fe}_{3} \mathrm{C}$ carbides precipitates were produced during isothermal annealing of Fe-C alloy at $473 \mathrm{~K}$ in oil.

\subsection{Apparatus set}

For the MBE measurements the samples were loaded using a torque machine with a constant $\mathrm{d} \tau / \mathrm{d} t$ rate and with a maximal level $2 \cdot 10^{-3} \mathrm{~N} \mathrm{~m} / \mathrm{s}$ of torque. The corresponding shear strain $\gamma$ was determined from light spot deflection. Internal friction measurements were performed by means of the inverted pendulum [3] with a vibration frequency of about $1 \mathrm{~Hz}$. The amplitude dependence of MIF was determined from the difference between the $Q^{-1}$ measured without and with saturated magnetic field, respectively. A magnetic field strengt up to $40 \mathrm{kA} / \mathrm{m}$ was provided by a solenoid. The loading machine and the MBE measuring electronic device have been made at Technical University of Gdanisk. Intensity of MBE signal induced in pick-up coil surrounding the sample was measured by means of analog integration of the rectified MBE voltage signal [11]. The total gain of the measuring system was about $120 \mathrm{~dB}$ and MBE voltage pulses have been amplified in frequency range from $0.5 \mathrm{kHz}$ up to $200 \mathrm{kHz}$.

\subsection{MBE strain dependence}

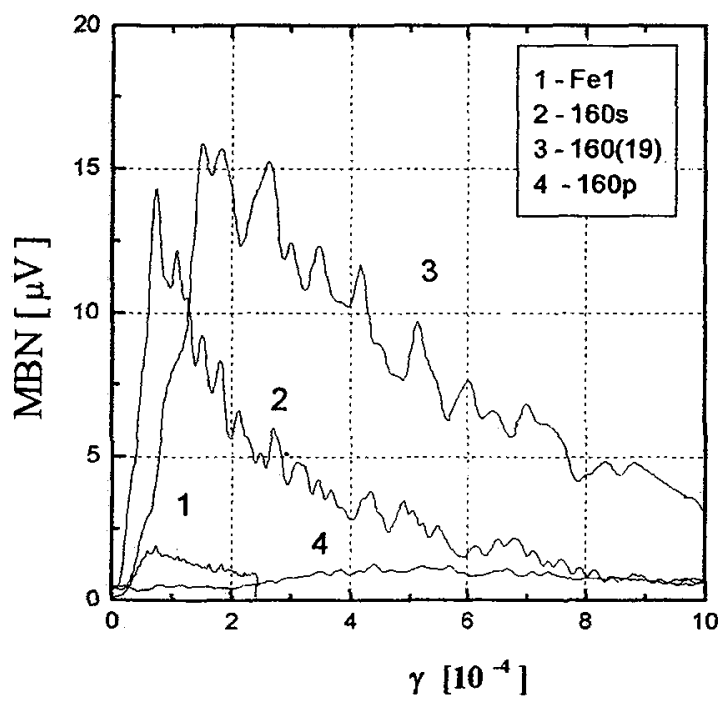

Fig. 1. MBE intensity plots recorded during "first load".
The "first load" method of MBE analysis means that the MBE intensity is recorded during the first loading of a demagnetized sample up to the given strain level $\gamma_{0}$. Fig. 1 presents four MBE intensity plots as recorded for the four tested sampies. There are shown results for pure $\mathrm{Fe}-$ 'Fel' and three states of Fe-C alloy: solid solution state - ' $160 \mathrm{~s}$ ', after annealing during $19 \mathrm{~min}$. - '160(19)' and precipitated state - ' 160 '. The MBE intensity level on Fig. 1 is related to the pick-up output voltage. The MBE plots reveal the maximum of MBE intensity which height and position is strongly related to the sample microstructure. The MBE intensity is very low for the 'Fel' sample. In the case of Fe-C alloy the MBE intensity is much higher and its maximum position shifts to higher strain levels during annealing. The MBE maximum is very broad and low for the sample '160p' in the precipitated state. 


\section{DISCUSSION}

The MBE signal indicates the change of the magnetic structure when external stress modifies the internal stress in the vicinity of "no-180" DW so that the DW is "depinned" and makes a Barkhausen jump. It means that the observed "first load" MBE stress dependence is strictly related to the internal stress distribution in equilibrium and thus can be used for microstructure state inspection. On the other hand the MBE intensity depicts magnetomechanical hysteresis process by means of individual events because each Barkhausen jump leads to energy losses due to eddy current and electromagnetic emission. In the case of cycling loading the magnetomechanical hysteresis process leads to macroscopic mechanical hysteresis loop which surface can be easily evaluated from stress dependence of MIF. Because it was revealed linear relation between integrals of MBE intensity and magnetomechanical losses as calculated for one cycle $[6,7]$ so we can conclude that the MBE intensity is closely related to the energy losse rate. In order to proof that conclusion we propose to use the "first load" MBE strain dependence to evaluate the MIF amplitude dependence. At first from that strain dependence of the MBE intensity there was calculated integral within strain range from $\gamma=0$ up to a given value $\gamma_{0}$. That integral value is postulated to be proportional to energy losses $\Delta \mathrm{W}$ in one cycle of mechanical loading with amplitude of strain equal to $\gamma_{0}$. From that integral one can evaluate the MIF strain dependence simply dividing $\Delta W$ by $\left(\gamma_{0}\right)^{2}$. Two results of such analysis are presented in Fig. 2 by line plots for the pure iron 'Fel' and in Fig. 3 for the partially annealed Fe-C alloy - '160(19)', respectively. In the figures there are also presented (by black points) the amplitude dependencies of MIF measured for the same samples by the pendulum. Both dependencies are normalized to the same peak level.

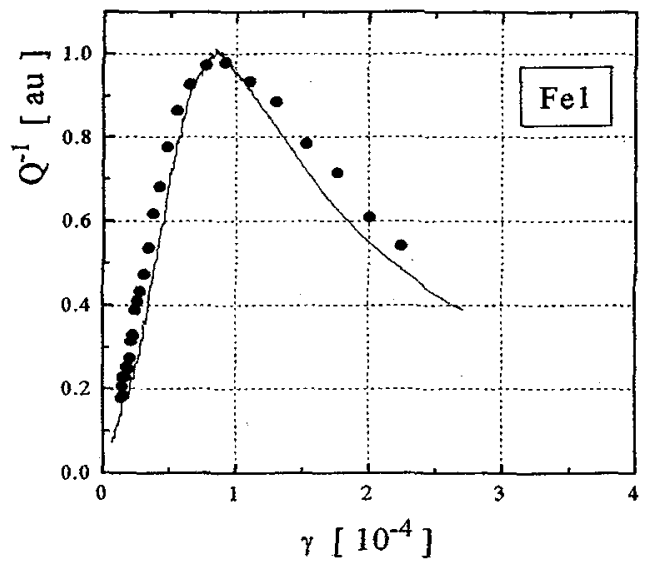

Fig. 2. Amplitude dependence of MIF for pure Fe: as measured (points) and evaluated from MBN (line)

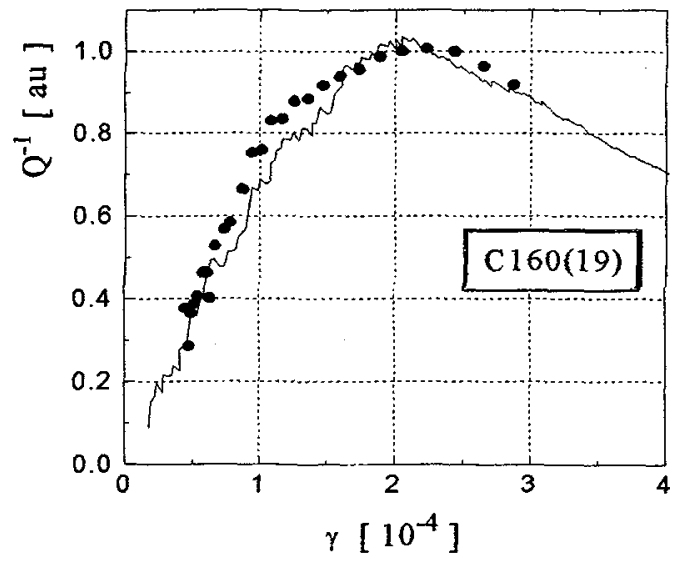

Fig. 3 Amplitude dependence of MF for Fe-C alloy: as measured (points) and evaluated from MBN (line)

The obtained correlation between both functions is very close. This remarkable result gives evidence that the proposed procedure is self consistent. It seems that the postulated linear relationship between MBE intensity and energy loss rate: $U(\gamma) \approx \mathrm{d} W / \mathrm{dt}$ is well confirmed. On the basis of this relation we propose a new way of evaluation of the internal strains distribution function $N(\gamma)$ using only the "first load" MBE function $U(\gamma)$. It can be done assuming - as in the magnetomechanical damping model [2] - that the energy loss rate $\mathrm{d} W / \mathrm{d} \sigma$ is proportional to the product of $N(\sigma)$ function and applied stress $\sigma$. In the case of shear stress load we replace simply a normal stress $\sigma$ by a shear stress $\tau$ or strain $\gamma$. The resulting relation between $\mathrm{MBE}$ intensity $\mathrm{U}(\gamma)$ and $N(\gamma)$ function is thus as follows: $U(\gamma) \approx \gamma \cdot N(\gamma)$. The $N(\gamma)$ dependence can be then evaluated simply by dividing $U(\gamma)$ by shear strain level $\gamma$. The results of such evaluation of the $N(\gamma)$ functions for the same four samples are shown in Fig. 4 . These functions are normalized setting the integrals to $\int N(\gamma) \mathrm{d} \gamma=1$. 


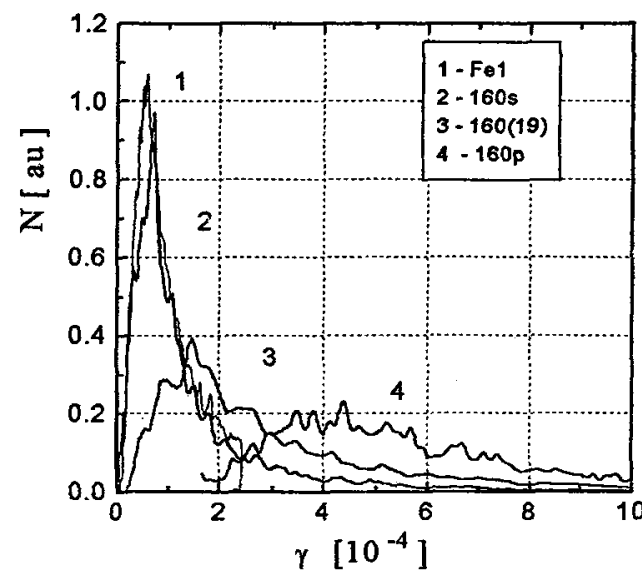

Fig. 4. Internal strains distribution functions $N(\gamma)$

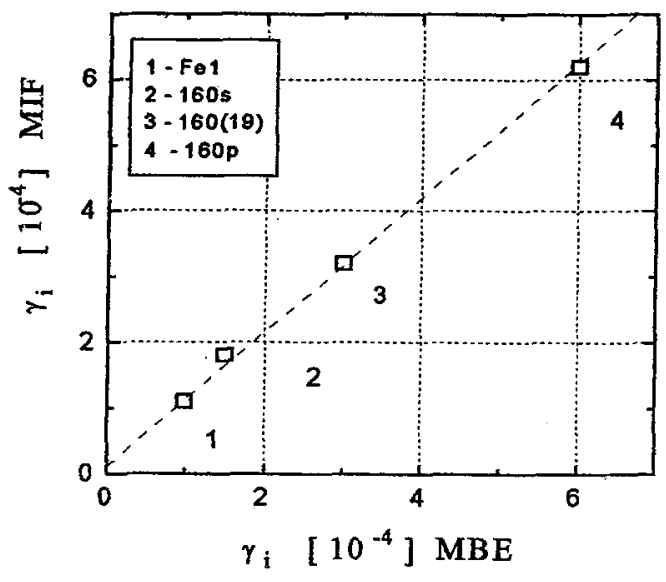

Fig. 5. Mean values of internal strains as calculated from MBE and MIF

From this function one can obtain the internal stress $N(\sigma)$ distribution function after scaling shear strain $\gamma$ to effective normal stress $\sigma$ using the known formula [2]: $\sigma \approx \sqrt{3} E \gamma$, where $E$ is Young's modulus. The obtained $N(\gamma)$ functions (in Fig. 4) reveal remarkable evolution of internal stresses in the tested samples. The functions $N(\sigma)$ can be used to calculation of the $\sigma_{\mathrm{i}}$ parameter values because $\sigma_{\mathrm{i}}=\int \sigma N(\sigma) \mathrm{d} \sigma$. Such calculations have been performed for $\gamma_{\mathrm{i}}$ parameter (equivalent to $\sigma_{\mathrm{i}}$ ) using the obtained $N(\gamma)$ functions. The resulting values of $\gamma_{i}$ parameter were compared with those which were evaluated from the MIF stress dependence using Birchak's and Smith model of magnetomechanical damping [2] and the first type of $N(\sigma)$ function: $N(\sigma) \approx \sigma \exp \left(-2 \sigma / \sigma_{\mathrm{i}}\right)$. The resulting linear relation between these two parameters is shown in Fig. 5. Such very good correlation confirms again validity of the proposed method of internal stress function evaluation from the "first load" MBE intensity function. There is also other important feature of the "first load" MBE function which one can conclude comparing Fig. 1 and Fig. 5: positions of the maximum of the $U(\gamma)$ function are very close to the evaluated levels of the $\gamma_{i}$ parameter. Such relationship can be thus used for a quick estimation of the $\sigma_{\mathrm{i}}$ parameter level.

\section{CONCLUSIONS}

The "first load" stress dependence of the MBE intensity provides essential information concerning magnetomechanical hysteresis process and can be used for direct evaluation of the internal stress distribution function $N(\sigma)$ as well for a quick estimation of the $\sigma_{\mathrm{i}}$ parameter level.

\section{References}

[1] Bozorth R.M., Feromagnetism, D. Van Nostrand Comp., New York, 1961.

[2] Birchak J.R, Smith G.W., J. Appl. Phys. 43 (1972) 1238-1245.

[3] Degauque J., Thése, Universite de Toulouse, 1977.

[4] Augustyniak B., Fantozzi G., Vannes A., J. de Phys. 46 (1985) C10-733.

[5] Augustyniak B., Vannes A., J. de Phys. 48 (1987) C8-407.

[6] Augustyniak B., Mater. Sc. Forum 119-121 (1993) 585-590.

[7] Augustyniak B, Chmielewski M., Chicois J., ICIFUAS -11, Poitiers (1996) Abstracts P5B-4.

[8] Augustyniak B., J. of Alloys and Comp.211-212 (1994) 563-567.

[9] Małkinski L., Kaczkowski K., Augustyniak B., J. of Magn. Magn. Mater., 112(1992) 323-324.

[10] Augustyniak B., Degauque J., J. of Magn. Magn. Mater., 140-144 (1995) 1837-1838.

[11] Sablik M.J., Augustyniak B., J. Appl. Phys. 79 (1996) 963-972. 